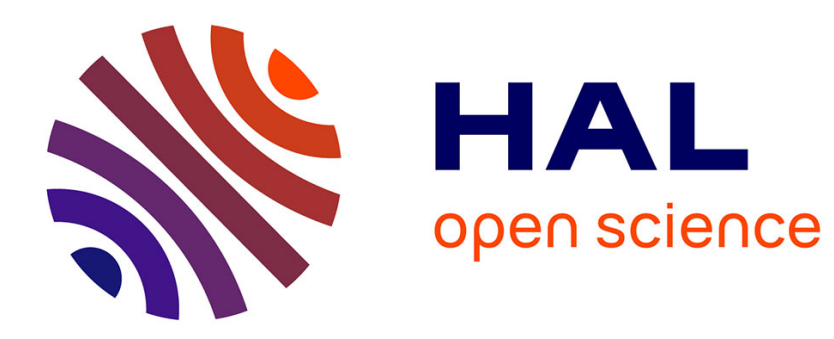

\title{
An evaluation of the number of Hamiltonian paths
}

Henri Orland, C. Itzykson, Cirano de Dominicis

\section{To cite this version:}

Henri Orland, C. Itzykson, Cirano de Dominicis. An evaluation of the number of Hamiltonian paths. Journal de Physique Lettres, 1985, 46 (8), pp.353-357. 10.1051/jphyslet:01985004608035300 . jpa00232523

\section{HAL Id: jpa-00232523 https://hal.science/jpa-00232523}

Submitted on 1 Jan 1985

HAL is a multi-disciplinary open access archive for the deposit and dissemination of scientific research documents, whether they are published or not. The documents may come from teaching and research institutions in France or abroad, or from public or private research centers.
L'archive ouverte pluridisciplinaire HAL, est destinée au dépôt et à la diffusion de documents scientifiques de niveau recherche, publiés ou non, émanant des établissements d'enseignement et de recherche français ou étrangers, des laboratoires publics ou privés. 
Classification

Physics Abstracts

$05.20-05.40-05.50$

\title{
An evaluation of the number of Hamiltonian paths
}

\author{
H. Orland, C. Itzykson and C. de Dominicis \\ Service de Physique Théorique, CEN Saclay, 91191 Gif-sur-Yvette Cedex, France
}

(Reçu le 30 novembre 1984, révisé le 18 janvier 1985, accepté le 22 février 1985)

Résumé. - Le nombre de chemins Hamiltoniens sur un réseau régulier de $N$ points, de coordination $q$, est de la forme $\omega_{\mathrm{H}}^{N}$ pour $N$ grand. Nous estimons $\omega_{\mathrm{H}} \sim q / e$ en accord surprenant avec les données dont on dispose en dimension deux.

\begin{abstract}
The number of Hamiltonian walks on a regular lattice of $N$ points, with coordination number $q$ is of the form $\omega_{\mathrm{H}}^{N}$ for $N \rightarrow \infty$. We obtain an estimate $\omega_{\mathrm{H}} \sim q / e$ in surprising agreement with available data in two dimensions.
\end{abstract}

Conformation of condensed polymer globules $[1,5]$ or collapsed polymer chains are often modelled as compactly packed self-avoiding walks. The importance of enumerating such compact walks in the theory of the glass transition of polymer melts has been much discussed [2, 5].

This is known as the problem of Hamiltonian paths, i.e. paths which visit each point once and only once. In this Letter we consider only closed paths on a regular lattice.

\section{Mean field.}

Let $\mathcal{N}_{\mathrm{H}}$ be the number of Hamiltonian paths, $N$ the number of sites, $q$ the coordination number. The quantity $\frac{1}{N} \log \mathcal{N}_{\mathbf{H}}$ has a limit, which defines $\omega_{\mathbf{H}}$ as :

$$
\log \omega_{\mathbf{H}}=\lim _{N \rightarrow+\infty} \frac{1}{N} \log \mathcal{N}_{\mathbf{H}} .
$$

Several mean-field theories exist for the evaluation of $\omega_{\mathbf{H}}$. A Flory-Huggins type of theory [3] yields $\omega_{\mathrm{H}}=\frac{q-1}{e}$ and Huggins [4] quotes $\omega_{\mathrm{H}}=\frac{q-1}{2}$. These numbers are off by $10 \%$ to $40 \%$ for small coordination numbers ( $q=3$ for the 2D Honeycomb lattice and $q=4$ for the 2D square lattice), but the main disadvantage of these mean-field theories is that they cannot be corrected in a systematic way.

In this Letter, we present a new mean field theory, which yields $\omega_{\mathrm{H}}=q / e$, in excellent agreement with numerical estimates, and show how systematic corrections to this result can be calculated. 
We start with the representation

$$
\mathcal{N}_{\mathrm{H}}=\lim _{n \rightarrow 0} \frac{1}{n} \frac{\int \prod_{r} \mathrm{~d} \varphi_{r} \exp \left(-\frac{1}{2} \sum_{r, r^{\prime}} \varphi_{r} \Delta_{r r^{\prime}}^{-1} \varphi_{r^{\prime}}\right) \prod_{r}\left(\frac{\varphi_{r}^{2}}{2}\right)}{\int \prod_{r} \mathrm{~d} \varphi_{r} \exp \left(-\frac{1}{2} \sum_{r, r^{\prime}} \boldsymbol{\varphi}_{r} \Delta_{r r^{\prime}}^{-1} \varphi_{r^{\prime}}\right)}
$$

with periodic boundary conditions on the lattice. Let us note that the nature of the boundary conditions is crucial in the enumeration of Hamiltonian paths (indeed, Gordon et al. [5] show that with certain boundary conditions, $\omega_{\mathrm{H}}=0$ for the 2D Honeycomb lattice).

The integration variable $\varphi_{r}$ is an $n$ component field attached to point $r$ of the lattice, and the matrix $\Delta_{r r^{\prime}}$ is equal to 1 if $r$ and $r^{\prime}$ are nearest neighbours, and zero otherwise.

The use of Wick's theorem on equation (2) leads to a summation over graphs which reproduce the closed and connected Hamiltonian paths in the limit $n \rightarrow 0$. The difficulty associated with the non positive definiteness of the matrix $\Delta_{r r^{\prime}}$ (indeed, $\Delta_{r r^{\prime}}$ is a traceless matrix) could have been removed by multiplying it by $i$, and taking absolute values of the integrals. Finally, the analytic continuation at $n=0$ is straightforward, since Carlson's theorem applies. Indeed, for any integer $n$, one has :

$$
\begin{aligned}
& 0<\frac{\int \prod_{r} \mathrm{~d} \varphi_{r} \exp \left(-\frac{1}{2} \sum_{r, r^{\prime}} \varphi_{r} \Delta_{r r^{\prime}}^{-1} \varphi_{r^{\prime}}\right) \prod_{r}\left(\frac{\varphi_{r}^{2}}{2}\right)}{\int \prod_{r} \mathrm{~d} \varphi_{r} \exp \left(-\frac{1}{2} \sum_{r, r^{\prime}} \boldsymbol{\varphi}_{r} \Delta_{r r^{\prime}}^{-1} \varphi_{r^{\prime}}\right)} \leqslant \frac{\int \mathrm{du} \mathrm{e}^{-\mathbf{a}^{2} / 2}\left(\frac{\mathbf{u}^{2}}{2}\right)^{N}}{\int \mathrm{du} \mathrm{e}^{-\mathbf{u}^{2} / 2}} \\
&=\frac{\Gamma(N+n / 2)}{\Gamma(n / 2)} \\
&{ }_{n \rightarrow \sim+\infty}\left(N+\frac{n}{2}\right)^{N} .
\end{aligned}
$$

For large $n$, the integral is bounded by a power of $n$, and thus its analytical continuation at $n=0$ is unique.

$\mathcal{N}_{\mathrm{H}}$ is calculated by using the saddle-point method on equation (2). To get a uniform saddlepoint, we look for an extremum of the function

$$
\omega\left(\varphi^{2}\right)=\frac{\varphi^{2}}{2} \mathrm{e}^{-\varphi^{2} / 2 \varphi}
$$

where we have used $\sum_{r^{\prime}} \Delta_{r r^{\prime}}^{-1}=\frac{1}{q}$. Let us note again that a uniform saddle-point exists only if one chooses periodic boundary conditions on the lattice.

The corresponding value of $\varphi^{2} / 2$ is $q$, and we obtain $\omega_{\mathrm{SP}}=q / e$. Since the degeneracy of this saddle-point is the surface of the $0(n)$ sphere, $S_{n}=\frac{2 \pi^{n / 2}}{\Gamma(n / 2)} \sim n$, we obtain

$$
\mathcal{N}_{\mathbf{H}}=\omega_{\mathrm{SP}}^{N} \text { and } \omega_{\mathbf{H}} \sim \omega_{\mathrm{SP}}=\frac{q}{e}\left({ }^{1}\right) .
$$

${ }^{1}$ ) We wish to thank one of our referees for calling our attention to the fact that this result can be found by taking the $T=0$ limit of equation (5) in reference [13]. 
Had we taken $n=1$, instead of the limit $n=0$, this would have counted the number $\mathcal{N}_{\mathrm{K}}$ of non-connected closed paths visiting all sites once and only once. If we define $\omega_{\mathrm{K}}$ by

$$
\log \omega_{\mathbf{K}}=\lim _{N \rightarrow+\infty} \frac{1}{N} \log \mathcal{N}_{\mathbf{K}}
$$

the same method yields

$$
\omega_{\mathrm{K}} \sim \frac{2 q}{e} .
$$

We thus expect $\omega_{\mathrm{K}} / \omega_{\mathrm{H}}$ to be close to 2 . The saddle-point method yields a mean-field approximation. In the last paragraph, we calculate fluctuations.

\section{2: Numerical estimates.}

It is instructive to compare these expressions to some known results. Gujrati and Goldstein [6] mention that on a two-dimensional square lattice, one has the bounds

$$
\omega_{\mathbf{H}}^{(\mathbf{M})} \leqslant \omega_{\mathbf{H}} \leqslant \omega_{\text {ice }}
$$

where $\omega_{\mathbf{H}}^{(\mathbf{M})}$ is the analog of $\omega_{\mathbf{H}}$ on a Manhattan lattice, (i.e. a lattice on which the vertical and horizontal bonds are oriented in alternating directions), which has been calculated exactly by Kasteleyn [7], whereas $\omega_{\text {ice }}$ is the exponential of the entropy of the 6-vertex model, given by Lieb [8]

$$
\omega_{\mathrm{H}}^{(\mathrm{M})}=1.338 \quad \omega_{\mathrm{ice}}=\left(\frac{4}{3}\right)^{3 / 2}=1.5396 .
$$

On such a lattice, $q=4$, and our estimate is

$$
\frac{q}{e}=1.4715
$$

Schmaltz, Hite and Klein [9] have calculated $\omega_{\mathrm{H}}$ using strip methods, and they give $\omega_{\mathrm{H}} \sim 1.472$, whereas Derrida [10] claims a value between 1.4725 and 1.473, i.e. larger than equation (9). On a hexagonal lattice with coordination 3, Derrida obtains $\omega_{\mathrm{H}} \sim 1.14$ whereas $3 / e \sim 1.10$.

We are not aware of any numerical data in dimension larger than 2 . On the same two-dimensional hexagonal lattice, the solution of the Ising model [11] at $\beta=i \pi / 2$ yields the exact value of $\omega_{\mathbf{K}}$ :

$$
\omega_{\mathrm{K}}=2 \exp \frac{1}{\pi} \int_{0}^{\pi / 3} \mathrm{~d} \theta \log (2 \cos \theta)
$$

so that the comparison with equation (6) yields :

$$
\frac{\omega_{\mathrm{K}}}{(6 / e)}=\frac{5 \sqrt{2}}{7} \exp \left[2 \sum_{1}^{\infty}\left(\frac{1}{2^{2 p+1}}-\frac{1}{6^{2 p+1}}\right) \frac{1}{2 p+1}(\zeta(2 p)-1)\right] .
$$

This ratio is equal to 1.065 .

The method used here can be generalized to the case where the path is not constrained to visit all points of the lattice, which is the case of polymers in a good solvent. Perhaps $q / e$ and $2 q / e$ are lower bounds to $\omega_{\mathbf{H}}$ and $\omega_{\mathbf{K}}$, but we have not succeeded in proving it. It is however worthwhile to note the extremely good agreement of this mean field theory with the available data. 


\section{3: Fluctuations.}

Finally, we show how to generate a $1 / q$ expansion of $\omega_{\mathrm{H}}$ around the mean field value.

The loop expansion around the mean field can be performed by shifting the integration field $\varphi_{r}$ by the mean field $\varphi$ in equation (2) in a standard way [12]. It is easily seen that this expansion yields a series for $\omega_{H}$ in powers of $1 / q$. This expansion is slightly complicated by the existence of Goldstone modes, which if not treated properly, give rise to infra-red divergencies. We illustrate this point by computing the one-loop correction (quadratic fluctuations). The longitudinal mode and the $(n-1)$ transverse modes have inverse propagators given respectively by

and

$$
G_{\mathrm{L}}^{-1}\left(r, r^{\prime}\right)=\Delta_{r r^{\prime}}^{-1}+\frac{1}{q} \delta_{r r^{\prime}}
$$

$$
G_{\mathrm{T}}^{-1}\left(r, r^{\prime}\right)=\Delta_{r r^{\prime}}^{-1}-\frac{1}{q} \delta_{r r^{\prime}}
$$

The identity

$$
\sum_{r^{\prime}} \Delta_{r r^{\prime}}^{-1}=\frac{1}{q}
$$

shows that there are $(n-1)$ transverse Goldstone modes, due to the $0(n)$ symmetry breaking.

In addition, if antiferromagnetic ordering is possible on the lattice (e.g. for square lattices, or hexagonal lattices) the longitudinal inverse propagator has a zero at $\mathbf{k}=(\pi, \ldots, \pi)$. This zero mode can also be interpreted as the Goldstone boson associated with a continuous symmetry of the mean-field $\varphi$. Indeed, we could have chosen the mean field to be equal to a constant $\varphi_{1}$ on one sublattice and $\varphi_{2}$ on the other sublattice. The mean field equation becomes

$$
\varphi_{1} \varphi_{2}=2 q
$$

which shows that the general solution can be parametrized as $\varphi=\sqrt{2 q} \lambda$ on one sublattice and $\varphi=\frac{\sqrt{2 q}}{\lambda}$ on the other sublattice, where $\lambda$ is any non zero number.

Having identified all the Goldstone modes, they can be projected out, using for instance the Fadeev-Popov method [12]. To first order the result is

$$
Z=\left(\frac{q}{e}\right)^{N} \exp \left[-\frac{1}{2} \log \operatorname{Det}\left(\frac{\delta_{r r^{\prime}}+\Delta_{r r^{\prime}} / q}{\delta_{r r^{\prime}}-\Delta_{r r^{\prime}} / q}\right)\right]
$$

where the Goldstone modes are removed from the determinants. By calculating these determinants in Fourier space, it is readily seen that they are equal, and thus :

$$
\omega_{\mathrm{H}}=\frac{q}{e}+O\left(\frac{1}{q^{2}}\right) .
$$

The vanishing of the quadratic fluctuations is perhaps responsible for the very good agreement of the mean-field results.

\section{Acknowledgments.}

We thank B. Derrida for providing us with unpublished numerical data, as well as J. des Cloizeaux who introduced us to the concept of subadditivity. 


\section{References}

[1] Nagle, F. F., Proc. R. Soc. A 337 (1974) 569.

Pechold, W. R. and Grossman, H. P., Faraday Disc. 68 (1979) 58.

Nagle, J. F., Gujrati, P. D., Goldstein, M., J. Phys. Chem. 88 (1984) 4599.

[2] Gujrati, P. D., J. Phys. A 13 (1980) L437.

FloRY, P. J., Proc. Natl. Acad. Sci. USA 79, 4510.

[3] Mears, P., Polymers : Structure and Bulk Properties (London, Van Nostrand) 1965.

VRIJ, A. and VAN DEN Esker, M. W. J., J. Chem. Soc. Faraday, Trans. II 68 (1972) 513.

[4] Huggins, M. L., Ann. N.Y. Acad. Sci. 4 (1942) 1.

[5] Gordon, M., Kapadia, P., Malakis, A., J. Phys. A 9 (1976) 751.

[6] Gujrati, P. D., Goldstein, M., J. Chem. Phys. 74 (1981) 2596 and references therein.

[7] Kasteleyn, P. W., Physica 29 (1962) 1329.

[8] LieB, E. H., Phys. Rev. Lett. 18 (1967) 692.

[9] Schmaltz, T. G., Hite, G. E., Klein, D. J., J. Phys. A 17 (1984) 445.

[10] DERRIDA, B., private communication.

[11] Houtappel, R. M. F., Physica 16 (1950) 425.

[12] ItZyKSON, C., ZuBer, J. B., Quantum Field Theory (Mc Graw-Hill International Book Company) 1980.

[13] Gujrati, P. D., Phys. Rev. B 25 (1982) 3381. 